\title{
Estudio de las propiedades psicométricas del Brief COPE para una muestra de mujeres uruguayas
}

\author{
Psychometric Properties of the Brief COPE in a Sample \\ of Uruguayan Women \\ Estudo das propriedades psicométricas do Brief COPE \\ para uma amostra de mulheres uruguaias
}

\author{
Micaela Reich ${ }^{*}$, César Daniel Costa-Ball ${ }^{* *}$, Eduardo Remor ${ }^{* * *}$ \\ ${ }^{*}$ Universidad ORT Uruguay, Montevideo, Uruguay. ${ }^{* *}$ Universidad Católica del Uruguay, \\ Montevideo, Uruguay. ${ }^{* * *}$ Universidade Federal do Rio Grande do Sul, Porto Alegre, Brasil.
}

Doi: http://dx.doi.org/10.12804/apl34.3.2016.13

\section{Resumen}

La escala Brief COPE (Carver, 1997) es la versión abreviada, ampliamente utilizada, del COPE Inventory (Carver, Scheier, \& Weintraub, 1989), que ha demostrado ser de utilidad para evaluar las estrategias de afrontamiento en investigaciones en salud. La versión en español del Brief COPE (Perczek, Carver, Price, \& Pozo-Kaderman, 2000) consta de 12 subescalas compuestas por dos ítems cada una. Se analizan las propiedades psicométricas del Brief COPE, en su versión en castellano, a partir del estudio en una muestra de 203 mujeres adultas pertenecientes a la población general uruguaya. El análisis factorial exploratorio, estudiando los ítems, indica la existencia de cuatro factores para la muestra estudiada. La agrupación resultó considerablemente coincidente con la reportada por los autores de la escala. La fiabilidad y las evidencias de validez obtenidas con el Brief COPE para la presente muestra avalan su uso en el ámbito de la investigación en población del sexo femenino en Uruguay.

Palabras clave: afrontamiento; estrés; análisis factorial; propiedades psicométricas.

\section{flbstract}

The Brief COPE (Carver, 1997) is the widely used brief version of the COPE inventory (Carver, Scheier, \& Weintraub, 1989), which has broadly proven to be a useful measure of coping strategies in health-related research. The Brief COPE-Spanish version (Perczek, Carver, Price, \& Pozo-Kaderman, 2000) consists of 12 subscales, each of them are composed by two items. Psychometric properties of the BriefCOPE Spanish version are reported, derived from a sample of 203 Uruguayan adult women. An Exploratory Factor Analysis conducted

* $\quad$ Micaela Reich, Instituto de Educación, Universidad ORT Uruguay, Montevideo, Uruguay; ${ }^{* *}$ César Daniel Costa-Ball, Facultad de Psicología, Universidad Católica del Uruguay, Montevideo, Uruguay; ${ }^{* * *}$ Eduardo Remor, Instituto de Psicología, Universidade Federal do Rio Grande do Sul, Porto Alegre, Brasil.

La correspondencia relacionada con este artículo debe ser dirigida a Micaela Reich. Ph.D., Instituto de Educación - Universidad ORT Uruguay, Cuareim 1451, 11100, Montevideo, Uruguay. Tel.: +598 2908 9324. Correo electrónico: mreich@uni.ort.edu.uy

Cómo citar este artículo: Reich, M., Costa-Ball, C. D., \& Remor, E. (2016). Estudio de las propiedades psicométricas del Brief COPE para una muestra de mujeres uruguayas. Avances en Psicología Latinoamericana, 34(3), 615-636. doi: http://dx.doi.org/10.12804/ap134.3.2016.13 
with the scale items yielded 4 factors for the current sample. The emerging factor structure was consistent with the one described by the scale's authors. The use of the Brief COPE for research purposes in Uruguayan female populations is supported by reliability and evidences of validity obtained in the studied sample.

Keywords: coping; stress; factorial analysis; psychometric properties.

\section{Resumo}

A escala Brief COPE (Carver, 1997) é a amplamente utilizada versão abreviada do COPE inventory (Carver, Scheier, \& Weintraub, 1989), que tem demonstrado ser de utilidade para avaliar as estratégias de afrontamento em pesquisas de saúde. O Brief COPE-versão em espanhol (Perczek, Carver, Price, \& Pozo-Kaderman, 2000) consta de 12 subescalas compostas por dois itens cada uma. Estudam-se as propriedades psicométricas do Brief COPE, na sua versão em castelhano a partir do estudo em uma amostra de 203 mulheres adultas pertencentes à população geral uruguaia. A Análise Fatorial Exploratória, analisando os itens, indica a existência de 4 fatores para a amostra estudada. A agrupação resultou consideravelmente coincidente com a reportada pelos autores da escala. Fiabilidade e evidências de validez obtidas com o Brief COPE para a presente amostra avalizam o seu uso no âmbito da investigação em população do sexo feminino no Uruguai.

Palavras-chave: afrontamento; stress; análise fatorial; propriedades psicométricas.

Las diferentes respuestas personales ante las situaciones estresoras (independientemente de la naturaleza de dichas situaciones) resultan de los esfuerzos individuales por hacer frente a la fuente de estrés, modificarla, o bien al regular sus respuestas emocionales ante ésta, dotándola de un significado diferente para reducir su grado de amenaza (Lazarus \& Folkman, 1984, 1986; Pearlin, 1989). Podemos denominar estrategias de afrontamiento a las conductas de las personas que buscan frenar, amortiguar y, de ser posible, neutralizar o eliminar los efectos de las situaciones estresoras. Según Lazarus (2000), el afrontamiento se define "como los esfuerzos cognitivos y conductuales en constante cambio para la resolución de demandas internas o externas específicas que son valoradas como impositivas o excesivas para los recursos de las personas. El afrontamiento es el esfuerzo por resolver el estrés psicológico" (p. 122).

El afrontamiento ha sido descrito y analizado conceptualmente de diversas formas. Una de las más habituales distinciones teóricas es la que diferencia las estrategias de los estilos de afrontamiento. El primer caso refiere a formas de afrontamiento al estrés más específicas, como llevar a cabo acciones concretas para modificar las condiciones o efectos del estresor, o buscar apoyo social (por ejemplo, Morrison \& Bennett, 2008; Pearlin \& Schooler, 1978; Sandín, 2003). Cuando esas estrategias de afrontamiento se utilizan de forma habitual para hacer frente a los problemas hablaríamos de estilos de afrontamiento (Thoits, 1995). Éstos constituyen dimensiones más amplias; se trata de tendencias disposicionales a llevar a cabo unas u otras estrategias de afrontamiento. Según Carver et al. (1989), existen disposiciones personales para responder al estrés con unas estrategias más que con otras. Así mismo, existen autores que consideran que algunas personas cuentan con mayor espectro de recursos de afrontamiento que otras. Los recursos de afrontamiento son características personales y de manejo social en los que se basa la persona para hacer frente a los estresores, como la percepción de control, la autoeficacia o el sentido del optimismo (por ejemplo, Morrison \& Bennett, 2008; Pearlin \& Schooler, 1978; Sandín, 2003). Un afrontamiento adecuado a una determinada situación conlleva mejores niveles de ajuste y adaptación a la situación (Lazarus, DeLongis, Folkman, \& Gruen, 1985).

El afrontamiento puede considerarse un ejemplo de fenómeno no directamente observable, que pretende medirse, y para lo que se utilizan aproxima- 
ciones indirectas. Como tal, su medición conlleva el uso de indicadores observables, como lo son las respuestas de una persona a un cuestionario, y la bondad de tal medición dependerá de las garantías científicas de dicho instrumento (Carretero-Dios \& Pérez, 2005). Ante esto, cabe señalar la vital importancia que reviste para la psicología el estudio de dichas garantías en los instrumentos empleados.

Entre los instrumentos de medida de afrontamiento más ampliamente utilizados encontramos: Ways of Coping Questionnaire en sus diferentes versiones (Folkman \& Lazarus, 1980; Folkman \& Lazarus, 1985; Folkman, Lazarus, Dunkel-Schetter, DeLongis, \& Gruen, 1986); Coping Strategies Scale (Beckham \& Adams, 1984); Multidimensional Coping Inventory (Endler \& Parker, 1990); Inventario COPE (Carver et al., 1989); y Brief COPE (Carver, 1997). A pesar de que las diversas medidas difieren entre sí en varios aspectos, todas ellas evalúan tanto respuestas de afrontamiento focalizadas en el problema, como aquellas dirigidas a aspectos de la situación estresora que no son la situación en sí misma. Así mismo, las medidas típicamente evalúan las respuestas de afrontamiento que podrían ser disfuncionales o adaptativas (Carver, 1997).

El Brief COPE (Carver, 1997) es una versión abreviada del COPE, diseñada teniendo en cuenta las apreciaciones efectuadas por pacientes/participantes respecto a la extensión y redundancia de la medida en su versión original. La versión final del Brief COPE surgió a partir tanto de análisis factoriales previos, como de la claridad, relevancia y significado otorgado a los ítems por parte de los participantes. Las subescalas del instrumento se componen de dos ítems cada una, seleccionados dependiendo de si presentaron elevada carga factorial en el factor adecuado en los análisis originales de Carver et al. (1989), o de la claridad de los ítems percibida por los participantes a través de la experiencia de campo de los autores con la escala completa. Carver (1997) plantea que el instrumento es apropiado para su uso en investigación sobre los modos de afrontamiento en el ámbito de la salud. Entendemos que éste proporciona una medida breve de afrontamiento, permitiendo interpretar las respuestas como eficaces (o adaptativas) o ineficaces (o desadaptativas) al asociarse a diferentes niveles en otras variables psicosociales o de resultado, y al tipo de estresor. El COPE y el Brief COPE han sido utilizados en numerosos estudios, fundamentalmente en el área de la salud, y en diversos países (aparte de Estados Unidos), siendo necesaria su adaptación a diferentes idiomas; por ejemplo: Italia (Sica, Novara, Dorz, \& Sanavio, 1997); Croacia (Hudek-Knezevic, Kardum, \& Vukmirovic, 1999); Estonia (Kallasmaa \& Pulver, 2000); Australia (Lyne \& Roger, 2000); Francia (Muller \& Spitz, 2003); España (Pardo, 2009) y México (Moran, Landero, \& González, 2010). Para esta versión algunas subescalas fueron renombradas al haber presentado dificultades en trabajos anteriores. Así, "reinterpretación positiva y crecimiento" se convirtió en "reinterpretación positiva (no crecimiento)"; "enfocarse y expresar las propias emociones" se convirtió en "expresión de emociones negativas"; "desvinculación mental" se transformó en "autodistracción". También se incluyó una nueva subescala que no formaba parte del inventario original — una medida de dos ítems de autoculpa-, ya que se encontró que esta respuesta mostró ser importante en trabajos anteriores (Carver, 1997).

El Brief COPE, en su versión en español (Perczek et al., 2000) consiste en 12 subescalas de dos ítems cada una, según lo expresado por los autores del instrumento a través de la página web de la División de Salud, Departamento de Psicología, Universidad de Miami (FL), de la que forma parte Charles S. Carver. En esta versión, se omitieron dos subescalas (autoculpa y utilización de apoyo instrumental), dado que no habían demostrado ser de utilidad en estudios conducidos utilizando Brief COPE, versión original (Perczek et al., 2000). El Brief COPE fue traducido al español para Estados Unidos por investigadores de la División de Salud, Departamento de Psicología, Universidad de Mia- 
mi (FL), teniendo en cuenta la equivalencia léxica y gramatical, así como su equivalencia conceptual y cultural. Además, se consideró el emparejamiento del contenido del ítem con la dimensión que se quiere evaluar, llegando a una versión de consenso. Después se aplicó la versión final a una muestra de participantes para que realizaran sugerencias sobre cómo percibían las preguntas y el formato de respuesta. La consigna de administración del instrumento informa al participante que le serán planteadas algunas maneras de enfrentarse y adaptarse a situaciones difíciles. Se le solicita luego que piense en una situación difícil que haya tenido que enfrentar en el último año, y cumplimente el cuestionario informando cómo se enfrentó y adaptó a esa situación difícil.

Diversos estudios describen los siguientes aspectos acerca del contenido y agrupación de las subescalas en tres factores:

1. Afrontamiento focalizado en el problema, que incluiría: afrontamiento activo, planificación, reinterpretación positiva y crecimiento personal, supresión de actividades distractoras, aceptación, y refrenar el afrontamiento (Carver et al., 1993; Lazarus \& Folkman, 1984).

2. Afrontamiento focalizado en la emoción, que incluiría: utilización de apoyo instrumental y emocional, y enfocarse y expresar las propias emociones (Carver et al., 1989; Admiraal, Korthagen, \& Wubbels, 2000; Folkman \& Lazarus, 1980).

3. Afrontamiento por evitación, que incluiría: distanciamiento conductual y mental, negación del evento estresante, y humor (Holahan, Holahan, Moos, Brennan, \& Schutte, 2005).

También ha sido propuesta la agrupación de las escalas en dos factores, incluyendo en uno de ellos las estrategias teóricamente adaptativas, y en otro aquellas consideradas desadaptativas. Según esta distinción, la utilización de apoyo social parece estar situado en medio de ambos grupos (Carver et al., 1989). Considerando las escalas que componen la versión abreviada en castellano del cuestionario, y con base en lo planteado por Carver (1993) y Carver et al. (1997), entre las estrategias adaptativas encontramos: afrontamiento activo, planificación, reinterpretación positiva, aceptación, y utilización de apoyo emocional; entre aquellas estrategias que se pueden considerar desadaptativas encontramos: negación, desvinculación comportamental, autodistracción/desvinculación mental, expresión de emociones negativas, religión, uso de sustancias.

Según Connor-Smith y Compas (2004), las respuestas de afrontamiento activo dirigidas hacia el estresor o hacia los pensamientos y emociones asociadas a éste daban lugar a un mejor ajuste. Contrariamente, las respuestas de evitación o negación, que implican distanciarse del estresor y los pensamientos y emociones asociados a éste, se encontraron relacionadas a un peor ajuste.

En el Brief COPE, según lo informado por Perczek et al. (2000), en general, la validez interna de las medidas en español es comparable a la de la versión en inglés.

Carver et al. (1989) hallaron asociaciones entre afrontamiento activo y planificación y optimismo, con autoestima, con personalidad resistente y con patrón de conducta tipo A. Así mismo, el afrontamiento activo se relacionó negativamente con la ansiedad-rasgo. Un patrón similar sigue reinterpretación positiva, excepto que no correlaciona con patrón de conducta tipo A. Negación y desvinculación comportamental se encontraron asociadas con la ansiedad-rasgo, y negativamente con optimismo, autoestima y personalidad resistente. La correlación de las distintas escalas del COPE con una escala de deseabilidad social encontrada no fue muy fuerte, siendo la mayor encontrada con uso de sustancias, con reinterpretación positiva y crecimiento, con desvinculación comportamental, y con expresión de emociones negativas.

Se han observado patrones similares entre el COPE situacional y el disposicional. Sin embargo, se observaron diferencias significativas en las 
medias de afrontamiento activo mayor en disposicional, utilización de apoyo instrumental mayor en disposicional, reinterpretación positiva y crecimiento mayor en disposicional, religión mayor en disposicional, y desvinculación mental mayor en disposicional (Carver et al., 1989).

Miyazaki, Bodenhorn, Zalaquett y Kok-Mun (2008) describen la estructura factorial del Brief COPE, encontrando siete factores entre las 14 subescalas que propone la teoría para la versión de la escala en inglés de 28 ítems. Por su parte, Morán et al. (2010) encontraron una estructura factorial de 11 factores entre los 14 sugeridos originalmente.

El objetivo del presente estudio fue analizar las propiedades psicométricas de la versión en español para Estados Unidos (Perczek et al., 2000) del inventario Brief COPE de Carver (1997), en una muestra de mujeres pertenecientes a la población general uruguaya. El trabajo propone la determinación de la estructura factorial y de las dimensiones básicas de la escala (aquellas que puedan explicar la mayoría de la varianza observada en las respuestas de las participantes) en la muestra estudiada. Se plantea observar si los ítems del instrumento pueden ser resumidos, y la medida en que existen aspectos comunes entre ellos. En este sentido, se consideró interesante estudiar el modo en que se agruparían los factores, bien con base en lo planteado en la teoría de sus autores originales (por subescalas), si surgirían grupos según estilos de afrontamiento (focalizado en el problema, focalizado en la emoción o por evitación), o bien si lo harían con base en la categorización teórico-clínica de estrategias adaptativas y desadaptativas.

\section{Método}

\section{Participantes}

Un total de 203 mujeres adultas uruguayas pertenecientes a la población general participaron en el presente estudio (ver detalles en tabla 1). Para que las mujeres pudieran formar parte, fueron considerados criterios de inclusión: (a) la participación voluntaria y anónima; (b) el nivel cultural que habilite a la comprensión de los cuestionarios; (c) ser mayor de 30 años y menor de 65. Han sido considerados criterios de exclusión: (a) el estar consumiendo medicación psicoactiva; (b) presentar cualquier desorden mental o cognitivo que pudiera dificultar o impedir cumplimentar los cuestionarios de autoinforme (el cuestionario de datos clínicos diseñado para el presente estudio incluye preguntas respecto a diagnósticos y tratamientos de salud mental recibidos); (c) presentar enfermedades crónicas.

Para efectos de evaluar los criterios de inclusión y exclusión mencionados, se utilizó el formulario de datos sociodemográficos, laborales y clínicos diseñado ad hoc para este estudio. A través de éste, la información ha sido filtrada para que la muestra cumpla con los criterios descritos.

Tabla 1

Características sociodemográficas y laborales más salientes

\begin{tabular}{lc}
\hline \multicolumn{1}{c}{ Variables } & $\begin{array}{r}\text { Mujeres saludables } \\
(N=203)\end{array}$ \\
\hline Edad (años) $M(S D)$ & $47,58(9,78)$ \\
\hline Lugar de residencia (\%): & \\
Capital & 83,6 \\
Ciudad pequeña & 13,8 \\
Otras ciudades pequeñas & 2,6 \\
\hline Estado civil (\%): & \\
Casadas o viviendo en pareja & 64,7 \\
Sin pareja (solteras, divorciadas, & \\
viudas) & 29,3 \\
\hline Situación laboral (\%): & \\
Activa & 92,2 \\
Ama de casa & 6,9 \\
Incapacidad laboral transitoria & - \\
Jubiladas o pensionistas & 6,8 \\
\hline Nivel de estudios (\%): & \\
Secundario o técnicos & 22,4 \\
Universitarios & 70,7 \\
Primaria & 6,9 \\
\hline & \\
\hline
\end{tabular}

\section{Instrumentos de medida}


Brief COPE (Carver, 1997), en su versión en español para Estados Unidos (Perczek et al., 2000), se trata de un instrumento de autoinforme que evalúa las estrategias de afrontamiento empleadas, $\mathrm{y}$ consta de 24 ítems con un formato de respuesta de escala tipo Likert de cuatro puntos. El Brief COPE, en su versión en español, está compuesto por las siguientes subescalas (descritas en mayor detalle en la introducción del presente trabajo): autodistracción/distanciamiento mental; afrontamiento activo; negación; uso de sustancias; utilización de apoyo emocional; desvinculación comportamental; expresión de emociones negativas; reinterpretación positiva; planificación; humor; aceptación; religión.

Adaptación lingüistica para Uruguay. Después del contacto y autorización del autor original de la escala (Charles Carver, University of Miami, FL) para el desarrollo del presente estudio, se llevó a cabo una adaptación lingüística de la escala, considerando la posibilidad de que los regionalismos locales sugirieran la conveniencia de modificar algún ítem o palabra. Dado que no existe ningún cuestionario desarrollado o adaptado para evaluar estrategias de afrontamiento en Uruguay, se decidió realizar la adaptación del Brief COPE, en su versión en español, por ser uno de los instrumentos más utilizados en estudios clínicos y en diferentes países para evaluar las estrategias de afrontamiento en poblaciones generales y clínicas. Se evaluó que el lenguaje fuera claro, que la redacción fuera adecuada y que los ítems del cuestionario fueran comprensibles. Se administró la escala a 20 mujeres uruguayas cuya lengua madre era el castellano uruguayo, que residían en Uruguay. Se evaluó la equivalencia conceptual y semántica por medio de una prueba piloto, solicitando feedback respecto a la comprensión de las preguntas y a posibles sugerencias de modificación. De este proceso no surgieron cambios a la medida. La muestra empleada en este procedimiento no ha sido incluida en el análisis de datos.
Encuesta de datos sociodemográficos. Las variables sociodemográficas, laborales y clínicas se evaluaron empleando un formulario diseñado a tales efectos. Incluyó información respecto a la edad, lugar de residencia, estado civil, profesión, situación laboral, nivel de estudios alcanzado, existencia de un diagnóstico de trastorno de salud mental, tratamiento(s) de salud mental recibidos.

\section{Procedimientos}

El reclutamiento de participantes en este estudio se llevó a cabo a través de un muestreo no probabilístico, por conveniencia, a partir de bases de datos proporcionadas por la Universidad Católica del Uruguay (UCU), y referidas por participantes ya reclutadas. La participación fue voluntaria y las mujeres no obtuvieron ninguna compensación por dicha participación. Se les instruyó respecto a cumplimentar las medidas de forma individual, a la confidencialidad de la información y a que no había respuestas catalogables como "buenas" o "malas", sino que se pretendía conocer la forma de responder de las personas uruguayas ante las situaciones de estrés. No se estableció un límite de tiempo, aunque se les mencionó el tiempo estimado de cumplimentación de los formularios. El tiempo medio empleado por participante fue de 15-20 minutos. Algunos de los factores que pueden haber incidido como variables extrañas al no ser controladas por los investigadores han sido, por ejemplo, la hora de cumplimentación de los formularios, o variables ambientales como la temperatura del salón. Inicialmente se obtuvo la aprobación del Comité de Ética de Investigación de la UCU. Se presentó el proyecto y protocolo de investigación por escrito, por medio de entrevistas a las autoridades de las diferentes facultades y unidades institucionales a las que se les solicitaría participación, explicando los aspectos más relevantes del estudio. Parte de la muestra estudiada (funcionarias de la UCU) estuvo compuesta por mujeres pertene- 
cientes a diferentes áreas de gestión: Contaduría, Secretaría, Administración, Recursos Humanos, y Limpieza; y de docencia (docentes de aula o de tiempo completo). A partir del contacto con las participantes potenciales para integrar la muestra, se les explicaron los aspectos más relevantes del estudio y se obtuvo el consentimiento informado. Una vez que las mujeres manifestaban por escrito su participación voluntaria, se realizó una única evaluación mediante cuestionarios de autoinforme. Previo al inicio de la etapa de administración de cuestionarios, se entrenó en el procedimiento a aplicar a una estudiante investigador, una egresada y 19 estudiantes de la Licenciatura en Psicología, quienes constituyeron el grupo de colaboradores honorarios en la implementación. El entrenamiento consistió de dos reuniones grupales y reuniones individuales en caso de estimarse necesario, en las que se explicó la administración, incluyendo consignas, consentimientos informados, materiales, grilla de horarios y lugares de administración, así como otros aspectos prácticos y de organización del trabajo.

\section{Análisis estadísticos}

El análisis de datos se realizó por medio de los programas SPSS 22.0 y FACTOR 9.3 (Lorenzo-Seva \& Ferrando, 2006). El uso del programa FACTOR para el análisis factorial exploratorio (AFE) se debe a la ventaja que ofrece de llevar a cabo el estudio mediante matrices de correlación policóricas. Éstas se usan generalmente en los casos en que los ítems presentan un formato de respuesta tipo Likert (Muthen \& Kaplan, 1992). A su vez, el programa FACTOR proporciona análisis que no están disponibles en el SPSS, como el análisis paralelo (AP) de Horn (1965). Según la guía práctica y revisada sobre el AFE de ítems de Lloret-Segura, Ferreres-Traver, Hernández-Baeza y Tomás-Marco (2014), es aconsejable el uso del programa FACTOR para ejecutar el AFE a través de matrices policóricas cuando el tipo de datos usados tiene formato Likert y no presenta distribuciones normales. Se condujo un AFE con el objetivo de estimar el ajuste del modelo a los datos. Se buscó mejorar la estimación y el ajuste de modelo (Meade \& Kroustalis, 2005).

Como método de estimación de factores se optó por utilizar el Unweighted Least Squares (ULS, mínimos cuadros no ponderados en español), teniendo en cuenta que los ítems son de tipo ordinales (tipo Likert), cuando se viola el supuesto de normalidad, este es uno de los métodos más empleados" (Ximénez \& García, 2005). Permite factorizar matrices en situaciones adversas, incluso con pocos casos y muchos ítems, y sin necesidad de hacer supuestos distribucionales.

En relación con el número de factores a retener, se aconseja emplear varios métodos a fin de tomar una decisión. Se optó por retener factores compuestos por un número mínimo de ítems entre tres y cuatro (Lloret-Segura Ferreres-Traver, Hernández-Baeza, \& Tomás-Marco, 2014) y con saturaciones por encima a 0,30 (Kline, 1994).

Se comprobó el grado de adecuación de los datos del AF a través del cálculo de la medida KMO de Kaiser (1970). Valores entre .70 - .79 se consideran suficientes, y satisfactorios los valores mayores a .80 (Lloret-Segura et al., 2014).

A su vez, se tuvo en cuenta la valoración de la validez de contenido para evaluar la idoneidad de la factorización alcanzada (Carretero-Dios \& Pérez, 2005).

Por último, el método de rotación sugerido y empleado para este estudio fue una rotación oblicua (Normalized Direct_Oblimin). El criterio de la normalización oblicua Oblimin hace uso de un valor delta para controlar el grado de correlación. Valores delta iguales a 0 proveen factores con las más altas correlaciones, valores mayores y negativos proporcionan factores muy poco correlacionados (Martínez-Arias, Hernández-Lloreda, \& Hernández-Lloreda, 2006). La rotación contribuye a la 
interpretación de la solución factorial sin alterar la situación relativa de las variables, ni el porcentaje de la varianza de cada variable que el capaz de explicar el total de factores (Pardo \& Ruiz, 2002).

Para la evaluación de ajuste para decidir si un modelo con una determinada cantidad de factores resulta apropiado, se evaluó el grado de ajuste del modelo a los datos. Para calcular el número posible de factores a extraer, se corrió el análisis paralelo implementado en el programa libre distribución FACTOR (Lorenzo-Seva \& Ferrando, 2006). Esta técnica consiste en representar un gráfico Scree Plot con los autovalores que resultarían si el conjunto de datos no pudiese representarse por factores comunes, es decir, si las correlaciones entre las variables se debieran completamente al error de muestreo (Martínez-Arias et al., 2006). Así mismo, se revisó el gráfico de sedimentación (Scree Test) de Cattell $(1966,1978)$ que designa el número de factores en el punto previo a aquel en el que la pendiente se hace casi cero, pudiéndose considerar residual, y se tomaron para la interpretación de las dimensiones subyacentes aquellos ítems con una carga factorial con saturación superior a 0,30 .

Junto al procedimiento anterior, utilizamos tres criterios distintos para analizar si las correlaciones residuales son cero; si es así, es porque se ha extraído un número de factores comunes adecuado, no debiendo quedar varianza común, por lo tanto las correlaciones de los residuos deberían ser cero. Con este objetivo, usamos la raíz cuadrática residual (RMCR) y el índice gamma o GFI (Lloret-Segura et al., 2014, p. 1 162). Se utilizó la raíz media cuadrática residual (RMCR), que indica la magnitud media de las correlaciones residuales. Valores menores o iguales a 0,05 son indicativos de que el ajuste del modelo es aceptable; según el criterio de Kelley (1935), el error típico de un coeficiente de correlación de cero en la población es aproximadamente $1 / \sqrt{ } \mathrm{N}$, donde $\mathrm{N}$ es el tamaño de la muestra. Si la RMCR se mueve en torno a este valor, se puede interpretar que el ajuste del modelo es aceptable. En segundo lugar, se tomó el índice GFI de Tanaka y Huba (1985). Éste es una medida de bondad de ajuste, indicando la proporción de covariación entre las variables explicadas por el modelo estudiado. Valores mayores a 0,95 evidencian un buen ajuste (Ferrando \& AnguianoCarrasco, 2010). Finalmente, se obtuvo el índice de consistencia interna, índice de fiabilidad estimada calculado por el programa FACTOR (LorenzoSeva \& Ferrando, 2006). Éste informa la fiabilidad de las puntuaciones factoriales; la medida en las puntuaciones de la escala son fiables considerando únicamente la varianza que los ítems tienen en común. Dicho procedimiento arroja valores más altos (en comparación con el método alpha de Cronbach implementado en el SPSS) al estar trabajando con ítems con formato de respuesta tipo Likert (datos categóricos u ordinales); generalmente los ítems presentan escasa varianza en común, redundando en índices de consistencia interna más bajos.

\section{Resultados}

Con base en lo sugerido por Carretero-Dios y Pérez (2007), a continuación se presentan los resultados del análisis estadístico de los ítems y factores subsiguientemente emergentes del AFE. Así mismo, se muestran evidencias empíricas de la estructura interna de la prueba, y los resultados de la estimación de su fiabilidad para la muestra del presente estudio.

\section{Análisis descriptivos del Brief-COPE}

Se analizaron estadísticos descriptivos (media, desviación típica, mínimo, máximo, Kolmogorov-Smirnov, asimetría y curtosis) de los 24 ítems del instrumento (tabla 2). Estos datos se vieron reflejados en los valores de los estadísticos descriptivos conducidos en las subescalas que agrupan los ítems referidos más arriba. 


\section{Análisis factorial exploratorio (AFE)}

Después de los estadísticos descriptivos, los siguientes datos analizados fueron los proporcionados por la matriz de correlaciones entre todas las variables (presentada en la tabla 3). Se considera de interés señalar que el AFE tiene un papel importante en la verificación de la validez de constructo o del significado de la variable medida (por ejemplo, Stapleton, 1997). Los resultados de los análisis estadísticos descriptivos (desvío típico, asimetría y curtosis) sugieren valores adecuados para la mayoría de ítems. Se presentaron problemas en dos ítems, que arrojaron alta la asimetría y varianza $(4,10)$ (ver tabla 2). Por lo tanto, usamos la matriz policórica y el método de extracción de factores ULS. La extracción de la estructura factorial latente indica o determina el número de factores que permiten explicar las correlaciones entre las variables (Martínez-Arias et al., 2006). Así mismo,

Tabla 2.

Estadísticos descriptivos para la muestra de mujeres saludables $(N=203)$

\begin{tabular}{|c|c|c|c|c|c|c|}
\hline Ítem/estilo/subescala & Asimetría & Curtosis & M & DT & Mín. & Máx. \\
\hline $1 \mathrm{AEV}$ autodistracción/distanciamiento mental & $-0,125$ & $-1,141$ & 2,621 & 1,04 & 1 & 4 \\
\hline 2 AFP afrontamiento activo & $-0,626$ & $-0,515$ & 2,966 & 0,95 & 1 & 4 \\
\hline 3 AEV negación & 1,062 & $-0,124$ & 1,744 & 0,98 & 1 & 4 \\
\hline $4 \mathrm{AEV}$ sustancias & 3,898 & 14,705 & 1,153 & 0,55 & 1 & 4 \\
\hline 5 AFEM utilización de apoyo emocional & $-0,334$ & $-1,270$ & 2,734 & 1,13 & 1 & 4 \\
\hline $6 \mathrm{AEV}$ desvinculación comportamental & 1,298 & 0,557 & 1,621 & 0,91 & 1 & 4 \\
\hline 7 AFP afrontamiento activo & $-0,767$ & $-0,474$ & 3,089 & 0,97 & 1 & 4 \\
\hline 8 AEV negación & 0,879 & $-0,502$ & 1,828 & 1,00 & 1 & 4 \\
\hline 9 AFEM expresión de emociones negativas & 0,047 & $-1,200$ & 2,310 & 1,02 & 1 & 4 \\
\hline $10 \mathrm{AEV}$ sustancias & 14,212 & 199,000 & 1,010 & 0,14 & 1 & 3 \\
\hline 11 AFP reinterpretación positiva & $-0,089$ & $-0,952$ & 2,680 & 0,95 & 1 & 4 \\
\hline 12 AFP planificación & $-0,495$ & $-0,474$ & 2,847 & 0,90 & 1 & 4 \\
\hline 13 AFEM utilización de apoyo emocional & $-0,489$ & $-0,853$ & 2,901 & 1,00 & 1 & 4 \\
\hline 14 AEV desvinculación comportamental & 1,133 & 0,060 & 1,719 & 0,98 & 1 & 4 \\
\hline 15 AFP reinterpretación positiva & $-0,274$ & $-0,736$ & 2,768 & 0,91 & 1 & 4 \\
\hline 16 AFEM humor & 0,143 & $-1,230$ & 2,305 & 1,06 & 1 & 4 \\
\hline $17 \mathrm{AEV}$ autodistracción/distanciamiento mental & $-0,016$ & $-0,835$ & 2,576 & 0,92 & 1 & 4 \\
\hline 18 AFEM aceptación & $-0,490$ & $-0,747$ & 2,985 & 0,93 & 1 & 4 \\
\hline 19 AFEM expresión de emociones negativas & $-0,248$ & $-0,862$ & 2,685 & 0,96 & 1 & 4 \\
\hline 20 AEV religión & 0,247 & $-1,201$ & 2,315 & 1,08 & 1 & 4 \\
\hline 21 AFEM aceptación & $-0,399$ & $-0,525$ & 2,833 & 0,88 & 1 & 4 \\
\hline 22 AFP planificación & $-0,556$ & $-0,645$ & 3,069 & 0,89 & 1 & 4 \\
\hline 23 AEV religión & 0,002 & $-1,263$ & 2,522 & 1,08 & 1 & 4 \\
\hline 24 AFEM humor & 0,540 & $-0,856$ & 2,030 & 1,00 & 1 & 4 \\
\hline
\end{tabular}


el AFE permite poner a prueba la dimensionalidad pretendida por el autor en un determinado instrumento (Nunnally, 1978). Por otro lado, cabe mencionar que el hecho de hacer un AFE no prueba la validez del constructo, y es impropio aducir que se ha validado un instrumento por el simple hecho de efectuar un análisis factorial (Morales-Vallejo, Urosa-Sanz, \& Blanco-Blanco, 2003).

Se utilizó el criterio de análisis factorial paralelo (AP) que determinó cuatro factores (además del RMSR y GFI), y se aceptó la solución que aportó la estructura conceptualmente más clara. El primer valor que aparece en este gráfico es el mayor de los posibles y, por tanto, el más idóneo para su análisis al ser capaz de explicar la cantidad más relevante de la varianza total (Pardo \& Ruiz, 2002). El corte en la tendencia descendente sirve de regla para la determinación del número óptimo de factores que deben estar presentes en la solución (Pardo \& Ruiz, 2002). Así, se llegó a lo que Nunnally (1978) llama composición factorial o estructura factorial de la escala para la muestra estudiada.

Entre los distintos análisis efectuados en la muestra, utilizando diferentes métodos de extracción y de rotación con base en los objetivos del presente estudio, se optó por analizar los datos por medio del método de extracción de ULS y rotación Normalized Direct Oblimin, con Kaiser (1974) como método de rotación. Se condujo un AFE con los 24 ítems de la escala.

Se efectuó un análisis factorial exploratorio mediante el programa FACTOR 9.3 (Lorenzo-Seva \& Ferrando, 2006) sobre la matriz de correlaciones policóricas, con el objetivo de encontrar la estructura factorial de la escala de Carver (1997). Esta matriz está indicada especialmente para escalas conformadas por ítems de tipo Likert (Freiberg Hoffmann, Stover, de la Iglesia, \& Fernández Liporace, 2013; Lloret-Segura et al., 2014; Muthen \& Kaplan, 1992).

Consecuentemente, se utilizó la matriz policórica con el método de extracción de factores ULS. Se obtuvo una adecuación de los datos al AF a través del índice de Kaiser-Mayer-Olkin (KMO) de adecuación muestral $(0,72)$; representando una adecuación suficiente, a su vez, la prueba de esfericidad de Bartlett fue significativa (Chi cuadrado $=1506,5 ; p<0,000)$. Del análisis del scree-test (Cattell, 1966) y de los resultados obtenidos con el método de análisis paralelo (Horn, 1965), se señaló la adecuación de una solución de cuatro factores.

Obtenida la solución factorial más adecuada, y con el objetivo de obtener una solución más simple, se utiliza el método se rotación Oblimin (Normalized Direct_Oblimin). Con los 24 ítems se mantuvo el primer ítem con saturación de 0,27 con el objetivo de no eliminar ningún ítem de la escala. La varianza total de la escala es de 53,5\% (factor $1=22,6 \%$, factor $2=14,4 \%$, factor $3=9,2 \% \mathrm{y}$ factor $4=7,2 \%$ ) (ver tabla 5).

El AFE conducido con las puntuaciones de los ítems que componen cada una de las subescalas del instrumento indicó que el valor correspondiente a la prueba de Kaiser-Meyer-Olkin (KMO) para la muestra fue de 0,702 y la prueba de esfericidad fue significativa $\left(\chi^{2}=506,251 ; g l=66 ; p=0,000\right)$. Estos resultados indican que los datos son adecuados para conducir el AF. El número de factores sugerido por el criterio de autovalor mayor a $1(53,54 \%$ de varianza explicada) fue de 4 .

En la tabla 3 se presentan las matrices de pesos factoriales, con los resultados del $\mathrm{AF}$ de primer orden efectuado con los ítems del instrumento.

A continuación se procede a la descripción e interpretación de los factores emergentes, analizando los resultados obtenidos a la luz de la teoría sobre los constructos implicados (Martínez-Arias et al., 2006). Los cuatro factores emergentes para la muestra se agrupan de la siguiente manera (se nombran ítems, subescalas y estilos de afrontamiento en cada caso): 1 autodistracción/distanciamiento mental AEV/15 reinterpretación positiva AFP/16 humor AFEM/17 autodistracción/distanciamiento mental AEV/19 expresión de emociones negativas AFEM/24 humor AFEM, por un lado; 3 negación $\mathrm{AEV} / 6$ desvinculación comportamental AEV/8 
Tabla 3.

Resumen de reactivos y cargas factoriales para una solución oblicua de cuatro factores para Brief COPE $(N=203)$

\begin{tabular}{|c|c|c|c|c|c|}
\hline Número & Reactivo & F1 & $\mathrm{F} 2$ & F3 & F4 \\
\hline 1. & $\begin{array}{l}\text { Yo me enfoqué en el trabajo u otras actividades para dis- } \\
\text { traer mi mente. }\end{array}$ & 0,27 & 0,17 & $-0,14$ & 0,06 \\
\hline 15. & Yo busqué algo bueno en lo que estaba pasando. & 0,36 & $-0,22$ & 0,07 & 0,32 \\
\hline 16. & Yo hice bromas acerca de esto. & 0,92 & $-0,07$ & $-0,02$ & $-0,22$ \\
\hline 17. & $\begin{array}{l}\text { Yo hice algo para pensar menos en esto, como ir al cine, } \\
\text { ver TV, etc. }\end{array}$ & 0,38 & $-0,01$ & $-0,01$ & 0,01 \\
\hline 19. & Yo expresé mis pensamientos negativos. & 0,34 & $-0,06$ & 0,04 & 0,23 \\
\hline 24. & Yo hice gracia de la situación. & 0,56 & 0,17 & 0,05 & $-0,13$ \\
\hline 3. & Yo me dije a mi misma esto no es real. & 0,07 & 0,71 & $-0,06$ & 0,11 \\
\hline 6. & Yo me di por vencida de tratar de lidiar con esto. & 0,15 & 0,68 & 0,16 & $-0,19$ \\
\hline 8. & Yo rehusé creer que esto hubiera pasado. & 0,02 & 0,88 & 0,02 & 0,15 \\
\hline 9. & $\begin{array}{l}\text { Yo dije cosas para dejar escapar mis sentimientos desagra- } \\
\text { dables. }\end{array}$ & 0,05 & 0,41 & $-0,05$ & 0,38 \\
\hline 14. & Yo dejé de hacerle frente a la situación en la que estaba. & 0,21 & 0,62 & 0,23 & $-0,22$ \\
\hline 18. & Yo acepté la realidad de que esto haya pasado. & 0,10 & $-0,54$ & 0,17 & 0,21 \\
\hline 21. & Yo aprendí a vivir con esto. & $-0,22$ & $-0,35$ & 0,20 & 0,24 \\
\hline 4. & Yo usé alcohol u otras drogas para sentirme mejor. & 0,13 & 0,23 & 0,39 & $-0,17$ \\
\hline 10. & $\begin{array}{l}\text { Yo usé alcohol u otras drogas para que me ayudaran a } \\
\text { pasar por esto. }\end{array}$ & 0,05 & 0,26 & 0,43 & $-0,33$ \\
\hline 20. & $\begin{array}{l}\text { Yo traté de encontrar apoyo en mi religión o mis creencias } \\
\text { espirituales. }\end{array}$ & $-0,03$ & $-0,08$ & 0,81 & 0,21 \\
\hline 23. & Yo recé o medité. & $-0,19$ & 0,14 & 0,67 & 0,28 \\
\hline 2. & $\begin{array}{l}\text { Yo concentré mis esfuerzos para hacer algo acerca de la } \\
\text { situación en la que estaba. }\end{array}$ & 0,03 & $-0,23$ & 0,03 & 0,64 \\
\hline 5. & Yo recibí apoyo emocional de otras personas. & $-0,06$ & 0,30 & $-0,07$ & 0,85 \\
\hline 7. & Yo tomé acción para poder mejorar la situación. & $-0,07$ & $-0,22$ & 0,06 & 0,66 \\
\hline 11. & $\begin{array}{l}\text { Yo traté de verlo con un enfoque distinto para que parecie- } \\
\text { ra más positivo. }\end{array}$ & 0,30 & $-0,07$ & 0,037 & 0,42 \\
\hline 12. & Yo traté de crear una estrategia para saber qué hacer. & 0,13 & $-0,05$ & 0,049 & 0,55 \\
\hline 13. & Yo recibí apoyo y comprensión de alguien. & $-0,09$ & 0,09 & 0,01 & 0,74 \\
\hline 22. & Yo pensé mucho cuáles eran los pasos a seguir. & $-0,08$ & $-0,10$ & 0,13 & 0,59 \\
\hline $\begin{array}{l}\text { Varianza explic } \\
(53,54 \%) \\
\text { GFI }=0,96\end{array}$ & & 22,63 & 14,46 & 9,24 & 7,19 \\
\hline
\end{tabular}

Nota: F1. Humor-evitativo; F2. Evitativo-emocional; F3. Evitativo-religioso; F4. Focalizado en el problema-utilización de apoyo social. 
negación AEV/9 expresión de emociones negativas AFEM/14 desvinculación comportamental AEV/-18 aceptación AFEM/-21 aceptación por otro; 4 uso de sustancias AEV/10 uso de sustancias $\mathrm{AEV} / 20$ religión $\mathrm{AEV} / 23$ religión $\mathrm{AEV}$ por otro; y finalmente 2 afrontamiento activo AFP $/ 5$ utilización de apoyo emocional AFEM/7 afrontamiento activo AFP/11 reinterpretación positiva AFP/12 planificación AFP/13 utilización de apoyo emocional AFEM/22 planificación AFP, por otro.

Se optó por denominar al primer factor emergente "humor-evitativo", dada su composición con base en ítems que incluyen la autodistracción y humor como forma de hacer frente a las situaciones de estrés. Se denominó al segundo factor "evitativo-emocional", dada su composición con base en ítems que indican el uso de la evitación y el afrontamiento emocional. El tercer factor se denominó "evitativo-religioso", al componerse de estrategias habitualmente consideradas y asociadas a menores niveles de bienestar, mayores niveles de distrés (Carver et al., 1993; Cohen, 2002; Epping-Jordan et al., 1999; Gilbar, 2005; Gilbar \& Hevroni, 2007; Hack y Degner, 2004; Low, Stanton, Thompson, Kwan, \& Ganz, 2006; Osowiecki \& Compas, 1999; Stanton \& Snider, 1993; Tacon, Caldera, \& Bell, 2001; Thomas y Marks, 1995), y utilización de la religión como forma de hacer frente al estrés. Por último, se estimó conveniente denominar "focalizado en el problema-utilización de apoyo social" al cuarto factor, dada su composición mayoritariamente de estrategias habitualmente asociadas a mayor bienestar psicosocial y menores niveles de distrés (David, Montgomery, \& Bovbjerg, 2006; Epping-Jordan et al., 1999; Gilbar, Or Han, \& Plivazky, 2005; Holland \& Holahan, 2003, Osowiecki \& Compas, 1999; Stanton, Danoff-Burg, \& Huggins, 2002), y a la vinculación social.

Respecto al primer factor emergente, podría resultar interesante dejar planteado el interrogante de si el humor (hacer bromas sobre el estresor o reírse de las situaciones estresantes, haciendo burlas de éstas) estaría formando parte ya sea de los esfuerzos personales por reinterpretar positivamente el estresor (estando este factor también integrado por un ítem de reinterpretación positiva), de estrategias de afrontamiento, de evitación cognitiva, se integraría a los intentos por buscar apoyo social, o se asociaría de manera alterna a estos tres estilos. La autodistracción/distanciamiento mental (distanciarse del estresor, concentrarse en otros proyectos intentando distraerse con otras actividades, para tratar de no concentrarse en el estresor) implica la evitación cognitiva del estresor.

El segundo factor emergente ha quedado integrado por negación (intentar rechazar la realidad del problema, lo que conlleva negar la realidad del suceso estresante), y desvinculación comportamental (reducir o renunciar al esfuerzo propio que se hace para afrontar o lidiar con el estresor, incluso renunciando al esfuerzo para lograr las metas con las cuales se interfiere al estresor), posiblemente indicando la confluencia de estrategias que conllevan a la evitación conductual, como cognitiva del estresor, aparentemente como estilo disposicional de afrontamiento. Un ítem de expresión de emociones negativas (aumento de la consciencia del propio malestar emocional, acompañado de una tendencia a expresar o descargar esos sentimientos) se agrupó junto con las anteriores. Las estrategias de afrontamiento que integran este factor parecen tener en común el hecho de que en la literatura se las ha asociado a mayores niveles de distrés (discutiéndose en estudios transversales que niveles mayores de distrés generarían un mayor uso de la estrategia de manifestar las emociones negativas, o bien si el hecho de expresar las propias emociones negativas - si bien posiblemente resulte efectivo como estrategia en caso de conseguir el apoyo social esperado a partir de ello- pudiera, al menos a corto plazo, generar mayores niveles de distrés al hacer foco en dichas emociones) (Culver, Arena, Wimberly, Antoni, \& Carver, 2004; Roussi, Krikeli, Hatzidimitriou, \& Koutri, 2007). La aceptación ha integrado también este factor, 
encontrándose vinculado consistentemente a menores niveles de distrés, mayor ajuste y bienestar psicosocial (Carver et al., 1993; Culver et al., 2002; Neipp, López-Roig, Terol, \& Pastor-Mira, 2008; Roussi et al., 2007; Stanton et al., 2002). Algunos autores sugieren que la aceptación de la situación personal podría ser un precedente necesario para desplegar un afrontamiento activo (Carver et al., 1993); pudiendo contribuir a la toma de decisiones adaptativas (Stanton y Snider, 1993).

El tercer factor, compuesto por uso de sustancias psicoactivas y religión, parece sugerir que el uso del rezo o la meditación como forma de afrontar los estresores en mujeres uruguayas se encuentra asociado a las conducta de consumo. Este último factor parece indicar en la dirección de que el uso de la religión como estrategia de afrontamiento podría ser desadaptativo para las mujeres que participaron en este estudio.

En el cuarto factor emergente se integran estrategias focalizadas en el problema, sumadas a la búsqueda y utilización de fuentes de apoyo social-emocional como forma de afrontar el estrés. Los resultados del presente estudio son consistentes con los informados por Carver (1997), que encontró una estructura factorial emergente en la cual, al igual que en Carver et al. (1989) y Perczek et al. (2000), afrontamiento activo y planificación cargaron en un mismo factor, también integrado por un ítem de reinterpretación positiva, según Carver (1997). En suma, si bien la estructura factorial emergente del Brief COPE, versión en español, para la muestra estudiada no fue perfecta, resultó marcadamente similar a la descrita por Carver et al. (1989) para la escala completa, Carver (1997) para la versión en inglés, y Perczek et al. (2000) para la versión en castellano. Los valores de $\alpha$ para la muestra fueron aceptables en la amplia mayoría se las subescalas, datos que resultaron considerablemente coincidentes con los descritos por Carver (1997). Cabe mencionar que el instrumento incluye diversos pares de opuestos entre sus subescalas ( $p$, aceptación y negación), según la asunción de que el afrontamiento que las personas hacen ante las situaciones estresoras a menudo alterna respuestas incompatibles entre sí, incluso a través de breves periodos de tiempo (Perczek et al., 2000). Este aspecto podría ser estudiado con mayor detenimiento en futuras investigaciones.

\section{Análisis de fiabilidad}

Considerando que las subescalas están formadas solamente por dos ítems, se incluye como información complementaria la correlación entre los ítems. Cabe señalar que los valores del $\alpha$ de Cronbach para la muestra de estudio son aceptables o buenos en la mayoría de las 12 subescalas propuestas por el modelo teórico del Brief COPE. Todos los valores de $\alpha$ para el total de participantes $(N=203)$ exceden el 0,50, considerado el mínimo aceptable (Nunna1ly, 1978), a excepción de la subescala expresión de emociones negativas $(0,47)$. De hecho, la mayoría de valores exceden 0,60 excepto expresión de emociones negativas, autodistracción/distanciamiento mental, y uso de sustancias-planificación arrojó un valor de 0,58. Estos datos son parcialmente coincidentes con los descritos por Carver (1997), estudio en el cual se encontró que los valores de $\alpha$ excedían el 0,60 a excepción de las subescalas de expresión de emociones negativas, aceptación y negación. La administración de la escala global en el total de participantes arrojó como puntuación media 2,39 $(D T=0,40)$, con un $\alpha$ de Cronbach global de 0,78 (ver tabla 4 ).

De este modo, los valores de $\alpha$ para las "reacciones de afrontamiento" (Carver, 1997), atendiendo a la estructura original, fueron para el total de participantes $(N=203) 0,78$ para el afrontamiento focalizado en el problema, 0,65 para el afrontamiento focalizado en la emoción, y 0,54 para el afrontamiento por evitación.

Por otro lado, los valores de $\alpha$ para el total de participantes en los cuatro factores emergentes en la muestra fueron: factor $1=0,86$; factor $2=0,89$; factor $3=0,82$; factor $4=0,88$. 
Tabla 4.

Alpha de Cronbach de las 12 subescalas y de los cuatro factores emergentes del Brief COPE, versión en castellano siguiendo el instrumento original $(N=203)$

\begin{tabular}{|c|c|c|c|}
\hline Brief COPE & Ítems & $\begin{array}{l}\text { Alpha de } \\
\text { Cronbach }\end{array}$ & $\begin{array}{l}\text { Estilo de } \\
\text { afrontamiento }\end{array}$ \\
\hline Afrontamiento activo & 2.7 & 0,73 & AFP \\
\hline Planificación & 12.22 & 0,58 & AFP \\
\hline Reinterpretación positiva & 11.15 & 0,69 & AFP \\
\hline Aceptación & 18.21 & 0,66 & AFEM \\
\hline Humor & 16.24 & 0,76 & AFEM \\
\hline Religión & 20.23 & 0,86 & $\mathrm{AEV}$ \\
\hline Utilización de apoyo emocional & 5.13 & 0,81 & AFEM \\
\hline $\begin{array}{l}\text { Utilización de apoyo instrumental (omitido, no demostró ser de } \\
\text { utilidad en estudios utilizando Brief COPE. versión original) }\end{array}$ & - & - & AFP \\
\hline Autodistracción/distanciamiento mental & 1. 17 & 0,51 & AEV \\
\hline Negación & 3.8 & 0,65 & AEV \\
\hline Expresión de emociones negativas & 9.19 & 0,47 & AFEM \\
\hline Uso de sustancias & 4. 10 & 0,50 & AEV \\
\hline Desvinculación comportamental & 6.14 & 0,71 & AEV \\
\hline $\begin{array}{l}\text { Autoculpa (omitido, no demostró ser de utilidad en estudios utili- } \\
\text { zando Brief COPE. versión original) } \\
\text { Factor } 1 \\
\text { Factor } 2 \\
\text { Factor } 3 \\
\text { Factor } 4\end{array}$ & - & $\begin{array}{c}- \\
0,86 \\
0,89 \\
0,82 \\
0,88\end{array}$ & AFEM \\
\hline
\end{tabular}

Nota: $\mathrm{AFP}=$ afrontamiento focalizado en el problema; $\mathrm{AFEM}=$ afrontamiento focalizado en la emoción; $\mathrm{AEV}=$ afrontamiento por evitación.

Tabla 5 .

Autovalores, porcentajes de varianza y porcentajes acumulativos para los factores del Brief COPE

\begin{tabular}{cccc}
\hline Factor & Autovalor & \% de la varianza & \% acumulativo \\
\hline 1 & 5,43 & 22,63 & 22,63 \\
\hline 2 & 3,47 & 14,46 & 37,09 \\
\hline 3 & 2,21 & 9,24 & 46,34 \\
\hline 4 & 1,72 & 7,19 & 53,54 \\
\hline
\end{tabular}

Los indicadores de evaluación de ajuste muestran que el modelo de cuatro factores resultante del análisis factorial exploratorio ajusta satisfactoriamente. En primer lugar, según los resultados obtenidos con la raíz media cuadrática residual (RMCR), se han obtenido valores próximos $(0,06)$ al valor de referencia $(0,05)$ propuesto por Harman (1976), y, a su vez, inferior al valor de referencia $(0,07)$ propuesto por Kelley $(1935)$, con una muestra de 203 participantes, $1 / \sqrt{ } \mathrm{N}=0,07$. Como nuestro RMCR se mueve por debajo de este valor, es indicativo de un buen ajuste. En segundo lugar, se utilizó el índice GFI, que es una medida de ajuste que se mueve entre 0 y 1.

Los resultados con este índice superan el valor de referencia $(0,95)$, evidenciando que nuestro modelo factorial se ajusta a los datos (ver tabla 3 ). 


\section{Análisis descriptivos de los factores emergentes}

Como se puede observar en la tabla 6 , los resultados de los análisis estadísticos descriptivos (desvío típico, asimetría y curtosis) sugieren valores adecuados para los factores.

Tabla 6.

Estadísticos descriptivos de los cuatro factores emergentes $(N=203)$

\begin{tabular}{lcccccc}
\hline Factores & Asimetría & Curtosis & M & DT & Mín. & Máx. \\
\hline Factor 1 & 0,21 & $-0,07$ & 14,98 & 3,37 & 6 & 24 \\
\hline Factor 2 & 0,17 & $-0,33$ & 15,03 & 3,30 & 8 & 24 \\
\hline Factor 3 & 0,23 & $-0,86$ & 7,00 & 2,13 & 4 & 13 \\
\hline Factor 4 & $-0,48$ & $-0,35$ & 20,28 & 4,60 & 7 & 28 \\
\hline
\end{tabular}

\section{Discusión}

Carretero-Dios y Pérez (2007) plantean una serie de directrices que guían el desarrollo del presente estudio. Entre ellas, ya incorporadas al trabajo, se encuentran la delimitación conceptual del constructo estudiado, y la búsqueda y provisión de información referida a la construcción y evaluación de los ítems. Así mismo, se tuvo en cuenta una serie de consideraciones sugeridas por Carretero-Dios y Pérez (2005), a fin de evaluar la aplicabilidad de las directrices que ellos mismos sugieren para conducir el presente estudio instrumental. Al tratarse de un cuestionario de autoinforme con una escala de respuesta tipo Likert, al tener la escala como objetivo de la evaluación de un constructo relacionado a la psicología clínica y de la salud, no pudiendo ser enmarcada en el campo de las aptitudes, y al tratarse de un instrumento que evalúa una cualidad latente del participante y las diferencias individuales en dicha cualidad, se consideró apropiado seguir las mencionadas directrices.

Carver (s.f.) informa en su página web de la Universidad de Miami que la estructura factorial del instrumento no es estable, no arroja un índice de afrontamiento a través de una puntuación "global", y que su administración en diferentes muestras arroja diferentes patrones de relación entre los reactivos. Así mismo, el autor señala que no hay recomendaciones particulares respecto a la manera indicada de generar un estilo de afrontamiento dominante para una determinada persona o muestra. De hecho, recomienda observar los resultados arrojados en cada subescala, buscando la relación de éstos con otras posibles variables de interés. Por lo tanto, para el presente trabajo se ha decidido reportar la estructura factorial subyacente a la muestra estudiada.

El análisis psicométrico del cuestionario administrado a la población escogida arroja saturaciones factoriales estadísticamente significativas ( $p=0,000)$ y considerablemente elevadas $(>0,30)$. La estructura factorial propuesta por los autores originales de la escala (Carver, 1997; Perczek et al., 2000) se replica considerablemente en la muestra de mujeres uruguayas de población general que participaron de este estudio. La consistencia interna de las subescalas y de los factores emergentes en la muestra es buena en la mayoría de los casos. En este sentido, al igual que en estudios anteriores (Carver, 1997; Moran et al., 2010), los ítems que forman las subescalas de afrontamiento activo y planificación forman un solo factor. Así mismo, los ítems de ambas subescalas se agrupan en un único factor en el AFE conducido con los ítems apareados en la muestra, junto con reinterpretación positiva, al igual que lo descrito por Carver (1997).

Para el presente estudio, se planteó el objetivo de analizar las propiedades psicométricas del inventario Brief COPE (Carver, 1997) en su versión en español (Perczek et al., 2000) en una muestra de mujeres uruguayas. En el proceso se observó que la medida no ajustaba según la teoría y la estructura factorial planteada por sus autores; a partir del AFE de primer orden se replica considerablemente la estructura factorial original en la muestra. 
Entendimos que resultaría conveniente utilizar la puntuación de los ítems que componen cada subescala, dado que se trata de indicadores de cada forma de afrontamiento que pudieran ser relativamente independientes entre sí.

Se examinó el modo en que se agruparon los factores para la muestra estudiada, y se observó que lo hacen parcialmente con base en lo planteado en la teoría de los autores originales (por subescalas); han surgido también grupos según estilos de afrontamiento (focalizado en el problema, focalizado en la emoción o por evitación), y con base en la categorización teórico-clínica de estrategias adaptativas y desadaptativas. Así, los cuatro factores emergentes a partir del AF de primer orden están compuestos respectivamente por: 1 autodistracción/distanciamiento mental $\mathrm{AEV} / 15$ reinterpretación positiva AFP/16 humor AFEM/17 autodistracción/distanciamiento mental AEV/19 expresión de emociones negativas AFEM/24 humor AFEM, por un lado; 3 negación AEV/6 desvinculación comportamental AEV/8 negación AEV/9 expresión de emociones negativas AFEM/14 desvinculación comportamental AEV/-18 aceptación AFEM/-21 aceptación por otro; 4 uso de sustancias AEV/10 uso de sustancias $\mathrm{AEV} / 20$ religión $\mathrm{AEV} / 23$ religión $\mathrm{AEV}$, por otro; y, finalmente, 2 afrontamiento activo AFP/5 utilización de apoyo emocional AFEM/7 afrontamiento activo AFP/11 reinterpretación positiva AFP/12 planificación AFP/13 utilización de apoyo emocional AFEM/22 planificación AFP, por otro.

Los resultados del presente estudio son comparables con los reportados por Carver (1997), que encontró una estructura factorial emergente en la cual, al igual que en Carver et al. (1989) y Perczek et al. (2000), afrontamiento activo y planificación cargaron en un mismo factor, también integrado por reinterpretación positiva.

En suma, si bien la estructura factorial emergente del Brief COPE, versión en español, para la muestra estudiada no fue perfecta, resultó marcadamente similar a la descrita por Carver et al. (1989),
Carver (1997) y Perczek et al. (2000). Los valores de $\alpha$ para la muestra fueron aceptables en la amplia mayoría de las subescalas, datos que resultaron considerablemente coincidentes con los descritos por Carver (1997).

El instrumento incluye diversos pares de opuestos entre sus subescalas, según la asunción de que el afrontamiento que las personas hacen ante las situaciones estresoras a menudo alterna respuestas incompatibles entre sí, incluso a través de breves periodos de tiempo (Perczek et al., 2000). Este aspecto podría ser estudiado con mayor detenimiento en futuras investigaciones.

Entendemos que es necesario contar con instrumentos válidos, breves y de fácil administración que evalúen las estrategias de afrontamiento de personas que integran la población general y con enfermedades crónicas (Ornelas-Mejorada et al., 2013), que permitan identificar y abordar de manera sistemática las asociaciones entre las respuestas de afrontamiento de las personas y los resultados en salud.

Consideramos importante mencionar el hecho de que no existe en la literatura una nomenclatura común, un consenso en la forma de llamar o de configurar las categorías teórico-conceptuales de las diferentes estrategias, estilos y recursos de afrontamiento. La manera en que las estrategias de afrontamiento son agrupadas en la configuración de constructos en los diversos instrumentos varía sustancialmente entre estudios. Ello conlleva el dificultoso análisis y la complicada comparación de resultados obtenidos entre estudios que han administrado diferentes medidas. Una crítica recurrentemente planteada respecto a los instrumentos de medida de afrontamiento versa entorno al interrogante de si las estrategias de afrontamiento tal y como son conceptualizadas en las medidas se encuentran relacionadas entre sí, y el modo en que se da dicha relación. El rango de diversidad en las categorías propuestas por la literatura varía, y va desde uno amplio y específico, hasta la más simple dicotomía entre las estrategias de afrontamiento focalizadas 
en la emoción versus estrategias de afrontamiento focalizadas en el problema (categorías a las que habitualmente se suma la agrupación de estrategias de afrontamiento por evitación, discutidas todas más arriba). El concepto de afrontamiento es dinámico, por lo que los valores presentados en este estudio a partir del análisis de la escala en la población estudiada deben ser tomados con precaución (Pais-Ribero \& Morais, 2010).

Queda planteado el asunto de que los niveles de distrés pueden no ser una vía apropiada para valorar los resultados del afrontamiento en términos de que éste sea adaptativo o disfuncional. Posiblemente una persona pueda juzgar una determinada estrategia de afrontamiento como efectiva, a pesar de que su uso le provoque mayor distrés. La investigación en psicología de la salud podría beneficiarse al evaluar el afrontamiento por medio de un abordaje más dinámico, que incluya la valoración de la efectividad del afrontamiento, la evaluación del grado de libertad ejercida por las personas ante el uso de las estrategias de afrontamiento, y la examinación de los cambios producidos en el uso de estrategias de afrontamiento para hacer frente a estresores de diversa índole.

En suma, los resultados del presente estudio indican que el Brief COPE en su versión en español es un instrumento válido para evaluar estrategias de afrontamiento en mujeres adultas que integran la población general uruguaya. No obstante, estos datos deben ser interpretados teniendo en cuenta determinadas limitaciones presentes en este estudio. El reclutamiento de participantes se llevó a cabo a través de un muestreo no probabilístico, por conveniencia. Ello ha condicionado la selección de la muestra analizada para este estudio y, por tanto, su alcance. Los resultados aquí presentados son aplicables y generalizables únicamente a la población adulta general de género femenino con buen nivel socioeconómico y cultural, residente en las ciudades de Montevideo y Paysandú en Uruguay.

Teniendo en cuenta las posibles limitaciones descritas, y a fin de obtener mayor apoyo a la fia- bilidad y evidencias de validez del Brief COPE, versión en español, para poblaciones uruguayas, las investigaciones subsiguientes deberían considerar los siguientes aspectos:

(i) Evaluar las propiedades psicométricas de la escala en la muestra. (ii) Calcular las correlaciones entre el BriefCOPE y otros instrumentos que evalúen constructos clínicamente relevantes (por ejemplo, depresión, estrés percibido, CVRS) - ello podría contribuir a identificar, interpretar y analizar adecuadamente las estrategias de afrontamiento adaptativas al estar asociadas a mejor ajuste, mayores niveles de bienestar y CVRS, y menores niveles de distrés-. (iii) Establecer correlaciones con medidas obtenidas por observadores externos de las estrategias empleadas (por ejemplo, parejas, pares), a fin de conocer la validez referida a un criterio externo. (iv) Estudiar la sensibilidad del cuestionario para examinar y detectar cambios a partir de programas de intervención psicosocial. (v) Conducir un estudio instrumental a mayor escala, en el que se realice un análisis factorial confirmatorio (AFC), administrando el Brief COPE, en su versión en español, a una muestra de población general más amplia y representativa de la población uruguaya, compuesta por participantes de ambos géneros. (vi) Evaluar la estabilidad en el tiempo de las puntuaciones a través de la confiabilidad test-retest, y su vinculación a otras variables psicosociales o de resultados.

En cuanto al AF de primer orden, los ítems se agrupan lógicamente en tipos de afrontamiento más generales, indicando cuatro factores. Entre ellos, se observa una agrupación sustentable desde el punto de vista teórico-clínico, en las escasas oportunidades en que determinados ítems teóricamente no asociados cargaron en forma conjunta en los factores. 
Consideramos relevante continuar trabajando en el estudio de las propiedades psicométricas y la estructura factorial de este instrumento. Siguiendo lo propuesto por Morales-Vallejo et al. (2003), cabe señalar que las decisiones sobre los ítems que constituirán la versión definitiva de una escala deben tomarse siempre atendiendo a consideraciones sustantivas, sumado a la valoración conjunta de toda la información cuantitativa disponible.

\section{Referencias}

Admiraal, W. F., Korthagen, F. A. J., \& Wubbels, T. (2000). Effects of student teachers' coping behaviour. British Journal of Educational Psychology, 70(1), 33-52. doi: 10.1348/000709900157958

Beckham, E. \& Adams, R. (1984). Coping behavior in depression: report on a new scale. $B e-$ havior Research and Therapy, 22, 71-5. doi: 10.1016/0005-7967(84)90034-2

Carretero-Dios, H. \& Pérez, C. (2007). Normas para el desarrollo y revisión de estudios instrumentales: consideraciones sobre la selección de tests en la investigación psicológica. International Journal of Clinical and Health Psychology, 7, 863-882.

Carretero-Dios, H. \& Pérez, C. (2005). Normas para el desarrollo y revisión de estudios instrumentales. International Journal of Clinical and Health Psychology, 5, 521-51.

Cattell, R. B. (1978). The scientific use of factor analysis. Nueva York: Plenum Press. doi: 10.1007/978-1-4684-2262-7

Cattell, R. B. (1966). The scree test for the number of factors. Multivariate Behavioral Research, 1(2), 245-276. doi: 10.1207/s15327906mbr0102_10

Carver, C. S. (s.f.). Recuperado de http://www.psy. miami.edu/faculty/ccarver/sclBrCOPE.html

Carver, C. S. (1997). You want to measure coping but your protocol's too long: Consider the Brief COPE. International Journal of Behavioral
Medicine, 4, 92-100. doi: 10.1207/s15327558ijbm0401_6

Carver, C. S., Pozo, C., Harris, S. D., Noriega, V., Scheier, M. F., Robinson, D. S. et al. (1993). How coping mediates the effect of optimism on distress: A study of women with early stage breast cancer. Journal of Personality and Social Psychology, 65, 375-390. doi: 10.1037/00223514.65.2.375

Carver, C. S., Scheier, M. F., \& Weintraub, J. K. (1989). Assessing coping strategies: A theoretically based approach. Journal of Personality and Social Psychology, 56, 267-83. doi: 10.1037/0022-3514.56.2.267

Cohen, M. (2002). Coping and emotional distress in primary and recurrent breast cancer patients. Journal of Clinical Psychology in Medical Settings, 9(3), 245-51. doi: 10.1023/A:1016007529278

Connor-Smith, J. K. \& Compas, B. E. (2004). Coping as a moderator of relations between reactivity to Interpersonal stress, health status, and internalizing problems. Cognitive Therapy and Research, 28, 347-68. doi: 10.1023/B:COTR.0000031806.25021.d5

Culver, J. L., Arena, P. L., Wimberly, S. R., Antoni, M. H., \& Carver, C. S. (2004). Coping among African-American, Hispanic, and non-Hispanic White women recently treated for early stage breast cancer. Psychology \& Health, 19(2), 157-166. doi: 10.1080/08870440310001652669

Culver, J. L., Arena, P. L., Antoni, M. H., \& Carver, C. S. (2002). Coping and distress among women under treatment for early stage breast cancer: comparing African-Americans, Hispanics, and non-Hispanics whites. Psycho-Oncology, 11, 495-504. doi: 10.1002/pon.615

David, D., Montgomery, G. H., \& Bovbjerg, D. H. (2006). Relations between coping responses and optimism-pessimism in predicting anticipatory psychological distress in surgical breast cancer patients. Personality and Individual Differences, 40, 203-213. doi: 10.1016/j.paid.2005.05.018 
Endler, N. S. \& Parker, J. D. A. (1990). Multidimensional assessment of coping: a critical evaluation. Journal of Personality and Social Psychology, 58(5), 844-854.

Epping-Jordan, J. E., Compas, B. E., Osowiecki, D. M., Oppedisano, G., Gerhardt, C. A., Primo, K. et al. (1999). Psychological adjustment in breast cancer: Process of emotional distress. Health Psychology, 18, 315-26. doi: 10.1037/02786133.18.4.315

Ferrando, P. J. \& Anguiano-Carrasco, C. (2010). El análisis factorial como técnica de investigación psicológica. Papeles del Psicólogo, 31(1), 18-33.

Folkman, S. \& Lazarus, R. S. (1985). If it changes it must be a process: study of emotion and coping during three stages of a college examination. Journal of Personality and Social Psychology, 48, 150-70. doi: 10.1037/0022-3514.48.1.150

Folkman, S. \& Lazarus, R. S. (1980). An analysis of coping in a middle-aged community sample. Journal of Health and Social Behavior, 21(3), 219-39. doi: 10.2307/2136617

Folkman, S., Lazarus, R. S., Dunkel-Schetter, C., DeLongis, A., \& Gruen, R. (1986). The dynamics of a stressful encounter: Cognitive appraisal, coping and encounter outcomes. Journal of Personality and Social Psychology, 50, 992-1003. doi: 10.1037/0022-3514.50.5.992

Freiberg Hoffmann, A., Stover, J. B., de la Iglesia, G., \& Fernández Liporace, M. (2013). Correlaciones policóricas y tetracóricas en estudios factoriales exploratorios y confirmatorios. Ciencia Psicológica, 7(2), 151-164.

Gilbar, O. \& Hevroni, A. (2007). Counterfactuals, coping strategies, and psychological distress among breast cancer patients. Anxiety, Stress, \& Coping, 20(4), 383-392. doi: $10.1080 / 10615800701384439$

Gilbar, O. (2005). Breast cancer: how do Israeli women cope? A cross-sectional sample. Families, Systems, \& Health, 23(2), 161-171. doi: 10.1037/1091-7527.23.2.161
Gilbar, O., Or Han, K., \& Plivazky, N. (2005). Mental adjustment, coping strategies, and psychological distress among end-stage renal disease patients. Journal of Psychosomatic Research, 58, 471476. doi: 10.1016/j.jpsychores.2005.01.004

Hack, T. F. \& Degner, L. F. (2004). Coping responses following breast cancer diagnosis predict psychological adjustment three years later. Psycho-Oncology, 13, 247-253. doi: 10.1002/ pon.739

Harman, H. H. (1976). Modern factor analysis. Chicago: University of Chicago Press.

Holahan, C. J., Holahan, C. K., Moos, R. H., Brennan, P. L., \& Schutte, K. K. (2005). Stress generation, avoidance coping, and depressive symptoms: A 10-year model. Journal of Consulting and Clinical Psychology, 73(4), 658-66. doi: 10.1037/0022-006X.73.4.658

Holland, K. \& Holahan C. K. (2003). The relation of social support and coping to positive adaptation to breast cancer. Psychology and Health, 18(1), 15-29.

Horn, J. L. (1965). A rationale and test for the number of factors in factor analysis. Psychometrika, 30, 179-185. doi: 10.1007/BF02289447

Hudek-Knezevic, J., Kardum, I., \& Vukmirovic, Z. (1999). The structure of coping styles: A comparative study of Croatian sample. European Journal of Personality, 13, 149-61. doi: 10.1002/(SICI)1099-0984(199903/04)13:2\%3C149::AID-PER326\%3E3.0.CO;2-Z

Kaiser, H. F. (1974). An index of factorial simplicity. Psychometrika, 39, 31-36. doi: 10.1007/ BF02291575 Kaiser, H. F. (1970). A second generation Little Jiffy. Psychometrika, 35, 401- 415. Kallasmaa, T. \& Pulver, A. (2000). The structure and properties of the Estonia COPE inventory. Personality and Individual Differences, 29, 881-94. doi: 10.1016/S01918869(99)00240-8

Kaiser, H. F. (1974). An index of factorial simplicity. Psychometrika, 39, 31-36. doi: 10.1007/ BF02291575 
Kallasmaa, T. \& Pulver, A. (2000). The structure and properties of the Estonia COPE inventory. Personality and Individual Differences, 29, 881-94. doi: 10.1016/S0191-8869(99)00240-8

Kelley, T. L. (1935). Essential traits of mental life. Cambridge: Harvard Univ. Press.

Kline, P. (1994). An easy guide to factor analysis. Newburry Park: Sage.

Lazarus, R. (2000). Estrés y emoción. Manejo e implicaciones en nuestra salud. Bilbao: Desclée De Brouwer.

Lazarus, R. S., DeLongis, A., Folkman, S., \& Gruen, R. (1985). Stress and adaptational outcomes: The problem of confounded measures. American Psychologist, 40, 770-779. doi: 10.1037/0003066X.40.7.770

Lazarus, R. S. \& Folkman, S. (1984). Stress, appraisal and coping. Nueva York: Springer Publishing Company.

Lazarus, R. S. \& Folkman, S. (1986). Estrés y procesos cognitivos. Barcelona: Martínez Roca.

Lloret-Segura, S., Ferreres-Traver, A., Hernández-Baeza, A., \& Tomás-Marco, I. (2014). El análisis factorial exploratorio de los ítems: una guía práctica, revisada y actualizada. Anales de Psicología, 30(3), 1 151-1 169. doi: 10.6018/ analesps.30.3.199361

Lorenzo-Seva, U. \& Ferrando, P. J. (2006). FACTOR: A computer program to fit the exploratory factor analysis model. Behavioral Research Methods, Instruments and Computers, 38, 88-91. doi: 10.3758/BF03192753

Lorenzo-Seva, U. \& Ferrando, P. J. (1 de febrero, 2013). Manual of the program FACTOR. Recuperado de http://psico.fcep.urv.es/utilitats/ factor/

Low, C. A., Stanton, A. L., Thompson, N., Kwan, L., $\&$ Ganz, P. A. (2006). Contextual life stress and coping strategies as predictors of adjustment to breast cancer survivorship. Annals of Behavioral Medicine, 32(3), 235-244. doi: 10.1207/ s15324796abm3203 10
Lyne, K. \& Roger, D. (2000). A psychometric reassessment of the COPE questionnaire. Personality and Individual Differences, 29, 321-235. doi: 10.1016/S0191-8869(99)00196-8

Martínez-Arias, M. R., Hernández-Lloreda, M. J., \& Hernández-Lloreda, M. V. (2006). Psicometría. Madrid: Alianza Editorial.

McCaul, K, Sandgren, A., King, B., O’Donnell, S., Branstetter, A., \& Foreman, G. (1999). Coping and adjustment to breast cancer. Psycho-Oncology, 8, 230-236. doi: 10.1002/(SICI)1099-1611(199905/06)8:3\%3C230::AID-PON374\%3E3.0.CO;2-\%23

Meade, A. W. \& Kroustalis, C. M. (2005). Problems with item parceling for confirmatory factor analysis tests of measurement invariance of factor loadings. Paper presented at the 20th Annual Conference of the Society for Industrial and Organizational Psychology, Los Angeles, CA.

Miyazaki, Y., Bodenhorn, N., Zalaquett, C., \& KokMun, N. (2008). Factorial structure of Brief COPE for international students attending U.S. colleges. College Student Journal, 42(3), 795806.

Morales-Vallejo, P., Urosa-Sanz, B., \& Blanco-Blanco, A. (2003). Construcción de escalas de actitudes tipo Likert. Cuadernos de estadística. Madrid: La Muralla, Hespérides.

Morán, C., Landero, R., \& González, M.T. (2010). COPE-28: un análisis psicométrico de la versión en español del Brief COPE. Universitas Psychologica, 9(2), 543-552.

Morrison, V. \& Bennett, P. (2008). Psicología de la salud. Madrid: Pearson Educación.

Muller, L. \& Spitz, E. (2003). Evaluation multi-dimensionelle du coping: Validation du Brief COPE sur une population francaise. L'Encephale, XXIX, 507-518.

Muthén, B. \& Kaplan, D. (1992). A comparison of some methodologies for the factor anlysis of non-normal likert variables: A note on the size of the model. British Journal of Mathemati- 
cal and Statistical Psychology, 45, 19-30. doi: 10.1111/j.2044-8317.1992.tb00975.x

Neipp, M. C., López-Roig, S., Terol, M. C., \& Pastor-Mira, M. A. (2008). Coping and adaptation in breast cancer patients at the follow-up stage. Ansiedad y Estrés, 14(1), 115-126.

Nunnally, J. C. (1978). Psychometric theory (2a ed.). Nueva York: McGraw-Hill.

Ornelas Mejorada, R. E., Tufiño Tufiño, M. A., Vite Sierra, A., Tena Guerrero, O., Riveros Rosas, A., \& Sánchez Sosa, J. J. (2013). Afrontamiento en pacientes con cáncer de mama en radioterapia: análisis de la Escala COPE Breve. Psicología y Salud, 23(1), 55-62.

Osowiecki, D. M. \& Compas, B. E. (1999). A prospective study of coping, perceived control, and psychological adaptation to breast cancer. $\mathrm{Cog}$ nitive Therapy and Research, 23(2), 169-80. doi: 10.1023/A:1018779228432

Pais-Ribeiro, J. L. \& Morais, R. (2010). Adaptação portuguesa da escala breve de coping resiliente. Psicologia, Saúde \& Doenças, 11(1), 5-13.

Pardo, J. (2009). Estrés y salud en estudiantes de profesiones asistenciales (Tesis doctoral no publicada. Universidad Autónoma de Madrid, Madrid, España).

Pardo, A. \& Ruiz, M. A. (2002). SPSS 11. Guía para el análisis de datos. Madrid: McGraw-Hill.

Pearlin, L. I. (1989). The sociological study of stress. Journal of Health and Social Behavior, 30, 24156. doi: $10.2307 / 2136956$

Pearlin, L. L. \& Schooler, C. (1978). The structure of coping. Journal of Health and Social Behavior, 19, 2-21. doi: 10.2307/2136319

Perczek, R., Carver, C. S., Price, A. A., \& Pozo-Kaderman, C. (2000). Coping, mood, and aspects of personality in Spanish translation and evidence of convergence with English versions. Journal of Personality Assessment, 74(1), 6387. doi: 10.1207/S15327752JPA740105

Roussi, P., Krikeli, V., Hatzidimitriou, C., \& Koutri, I. (2007). Patterns of coping, flexibility in coping and psychological distress in women diagnosed with breast cancer. Cognitive Therapy and Research, 31, 97-109. doi: 10.1007/ s10608-006-9110-1

Sandín, B. (2003). El estrés: un análisis basado en el papel de los factores sociales. Revista Internacional de Psicología Clínica y de la Salud, 3(1), 141-57.

Sica, C., Novara, C., Dorz, S., \& Sanavio, E. (1997). Coping strategies: Evidence for cross-cultural differences? A preliminary study with the Italian version of coping orientations to problems experienced (COPE). Personality and Individual Differences, 23(6), 1026-1029. doi: 10.1016/ S0191-8869(97)00112-8

Stanton, A. L., Danoff-Burg, S., \& Huggins, M. E. (2002).The first year after breast cancer diagnosis: hope and coping strategies as predictors of adjustment. Psycho-Oncology, 11, 93-102. doi: 10.1002/pon.574

Stanton, A. L. \& Snider, P. R. (1993). Coping with breast cancer diagnosis: A prospective study. Health Psychology, 12, 16-23. doi: 10.1037/0278-6133.12.1.16

Stapleton, C. D. (1997). Basic concepts in Exploratory Factor Analysis (EFA) as a tool to evaluate score validity: A right-brained approach. Paper presented at the annual meeting of the Southwest Educational Research Association, Austin. Recuperado de http://ericae.net/ft/tamu/ Efa.htm

Tacon, A., Caldera, Y., \& Bell, N. (2001). Attachment style, emotional control, and breast cancer. $\mathrm{Fa}$ milies, Systems \& Health, 19(3), 319-326. doi: 10.1037/h0089455

Tanaka, J. S. \& Huba, G. J. (1985). A fit index for covariance structure models under arbitrary GLS estimation. British Journal of Mathematical and Statistical, 38, 197-201. doi: 10.1111/j.20448317.1985.tb00834.x

Thoits, P. A. (1995). Stress, coping and social support processes: Where are we? What next? Journal of Health and Social Behavior, número extra, 53-79. doi: 10.2307/2626957 
Thomas, S. F. \& Marks, D. F. (1995). The measurement of coping in breast cancer patients. Psycho-Oncology, 4, 231-237. doi: 10.1002/ pon.2960040309

Ximénez, M. C. \& García, A. G. (2005). Comparación de los métodos de estimación de máxima verosimilitud y mínimos cuadrados no ponderados en el análisis factorial confirmatorio. Psicothema, 17(3), 528-535.

Fecha de recepción: mayo 7, 2014

Fecha de aceptación: junio 30, 2014 